\title{
On the periodic variations of geomagnetic activity indices Ap and ap
}

\author{
H. Schreiber \\ Institut für Geophysik der Universität Göttingen, Herzberger Landstraße 180, D-37075 Göttingen, Germany \\ Fax: + 49551 397459; e-mail: hschrei@willi.uni-geophys.gwdg.de
}

Received: 10 June 1996 / Revised: 26 November 1997 / Accepted: 2 December 1997

\begin{abstract}
Yearly averages of geomagnetic activity indices Ap for the years 1967-1984 are compared to the respective averages of $v^{2} \cdot B_{s}$, where $v$ is the solar wind velocity and $B_{s}$ is the southward interplanetary magnetic field (IMF) component. The correlation of both quantities is known to be rather good. Comparing the averages of Ap with $v^{2}$ and $B_{s}$ separately we find that, during the declining phase of the solar cycle, $v^{2}$ and during the ascending phase $B_{s}$ have more influence on Ap. According to this observation (using Fourier spectral analysis) the semiannual and 27 days, Ap variations for the years 1932-1993 were analysed separately for years before and after sunspot minima. Only those time-intervals before sunspot minima with a significant 27-day recurrent period of the IMF sector structure and those intervals after sunspot minima with a significant 28-28.5-day recurrent period of the sector structure were used. The averaged spectra of the two Ap data sets clearly show a period of 27 days before and a period of 28-29 days after sunspot minimum. Moreover, the phase of the average semiannual wave of Ap is significantly different for the two groups of data: the Ap variation maximizes near the equinoxes during the declining phase of the sunspot cycle and near the beginning of April and October during the ascending phase of the sunspot cycle, as predicted by the RussellMcPherron (R-M) mechanism. Analysing the daily variation of ap in an analogue manner, the same equinoctial and R-M mechanisms are seen, suggesting that during phases of the solar cycle, when ap depends more on the IMF- $B_{s}$ component, the R-M mechanism is predominant, whereas during phases when ap increases as $v$ increases the equinoctial mechanism is more likely to be effective.
\end{abstract}

Key words. Interplanetary physics $\cdot$ Magnetic fields $\cdot$ Solar wind plasma $\cdot$ Solar wind - magnetosphere interaction

\section{Introduction}

Geomagnetic activity is generated by the interaction of the solar wind with the magnetosphere in connection with energy and mass transfer. Since the first in situ observations of the solar wind, numerous studies have been carried out to find quantitative relations between the parameters of the solar wind and its variability with geomagnetic indices. Soon thereafter, the solar wind velocity $v$ and the southward component $B_{s}$ of the interplanetary magnetic field IMF were realized to be the essential quantities causing geomagnetic activity (e.g. Hirshberg and Colburn, 1969; Arnoldy, 1971); other parameters (such as plasma density or temperature) are less important. Sources of high solar wind speed and associated strong southward IMF, generating increased geomagnetic activity, are found in coronal holes, fast coronal mass ejections (CMEs), and interaction regions between slow and fast solar wind (e.g. Crooker and Cliver, 1994, and references therein; Lindsay et al., 1995). Studies relating these solar wind characteristics with geomagnetic activity may reveal the physics of the interaction of solar and magnetospheric plasmas.

Most observations suggest that the solar wind energy is transferred to the magnetosphere by the reconnection process at the dayside magnetopause, where oppositely directed southward IMF and northward magnetospheric magnetic field lines merge (Dungey, 1961). Theoretical models of the merging process have been described (e.g. Petschek, 1964; Vasilyunas, 1975); however, not all details of the physics are yet understood (Dungey, 1994), and still other energy transfer mechanisms such as viscous interaction and Kelvin-Helmholtz wave instabilities at the flanks of the magnetosphere are discussed [see the comprehensive overview of Crooker and Siscoe (1986) and references therein]. 
The question of the physics governing the relation of the solar wind with geomagnetic activity may be investigated using synoptic observations of single events or by statistical analysis of time-series of the essential quantities. Here we follow the latter method, as have many others before, and study the periodic variations in the sun-earth-system: the 11-year solar cycle period, the semiannual and 27-day period, as well as the diurnal variation. We will show that in all these periodicities, not only that the field line merging or reconnection process is visible, but also (especially at particular phases of the solar cycle) that other mechanisms may be effective.

\section{The analysis of the periodic variations}

\subsection{The solar cycle periodic variations of $A p$}

To study the longer-period variations we use the Ap indices as daily averages of ap. The 11-year periodicity of geomagnetic activity runs approximately parallel to the cycle of sunspot number $R$; however, it is known that there are significant differences in the two cycles: geomagnetic activity has, for example, two peaks, one near sunspot maximum and the other in the declining phase of sunspots. These two peaks are seen to be particularly significant in the distribution of geomagnetic storms (Gonzalez et al., 1990). In general, the activity minimum appears 1 or 2 years after the sunspot minimum.

Spectral analyses of $R$ and Ap reveal a pronounced peak in the spectral power of $R$ at periods of 10.2-10.4 years, whereas Ap shows no comparable maximum (Fraser-Smith, 1972; Gonzalez et al., 1993). Our Fourier spectral analysis of the time-series of Ap and $R$ 19331993 gave no new results in the 11-year spectral range and are therefore not shown.

In Fig. 1 yearly averages of some quantities, regarding the following considerations, are shown for the years 1968-1984/1986. In the upper part, $\mathrm{Ap}$ and $v^{2} B_{s} / 10^{5}$ have comparable scales, where $v^{2}$ and $B_{s}$ are, respectively, the yearly averages of the squares of hourly values of the solar wind velocity $v$ in $\mathrm{km} / \mathrm{s}$, and the southward component $B_{s}$ of the IMF in nT and in solar magnetospheric coordinates. The hourly mean values of the interplanetary data are taken from the "OMNI" data base compiled by King $(1977,1986)$ and are available on CD-ROM of the National Geophysical Data Center, (NOAA/NESDIS/NGDC) Boulder, USA. The yearly $B_{s}$ values are computed from the hourly IMF- $B_{z}$ values by setting positive (northward directed) hourly $B_{z}$ averages equal to zero. In the lower part of Fig. 1 the respective yearly averages of $v^{2}$ and $B_{s}$ are shown separately. In the upper part there is a good correlation of the two curves (correlation coefficient $r=0.92$ ). This is expected, since most correlative studies resulted in best correlation between geomagnetic activity and $v^{2} B_{S}$ (see e.g. Crooker and Siscoe, 1986, and references therein). The lower part of Fig. 1, however, shows a variable influence of $v^{2}$ and $B_{s}$ on the activity in

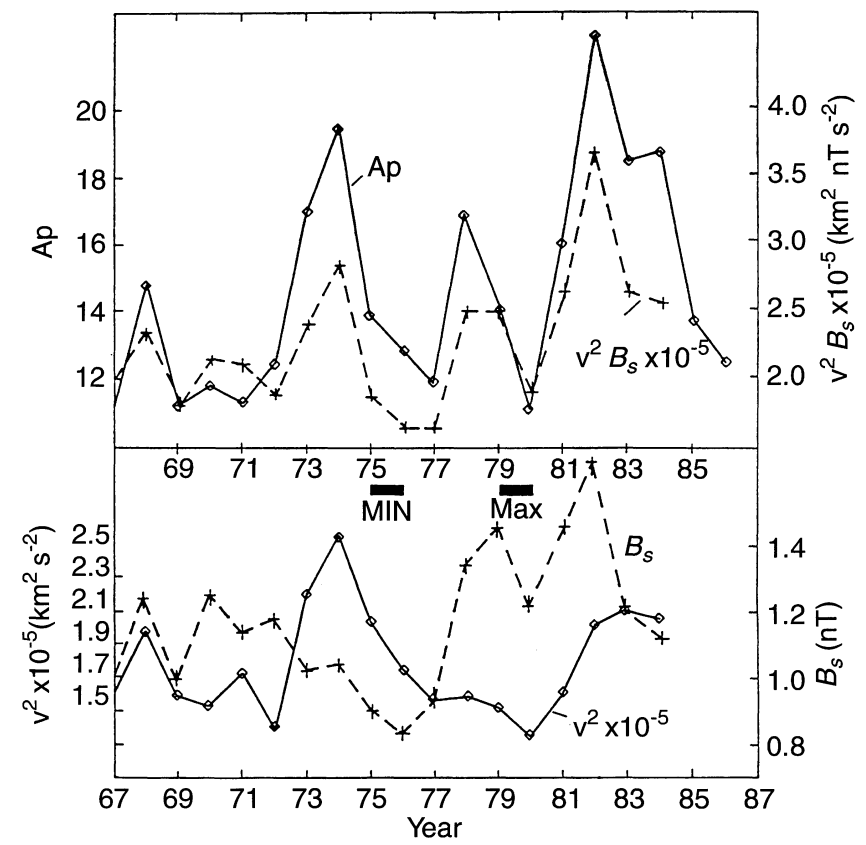

Fig. 1. Yearly averages of Ap and $v^{2} B_{s} / 10^{5}$ for the years 1967-1984 (to 1986 for Ap) upper part, and yearly averages of the southward IMF component $B_{s}$ and $v^{2} / 10^{5}$ lower part; maximum and minimum years of sunspot cycle 21 are indicated

the course of the sunspot cycle; during the declining phase in the years 1974-1976 increasing $v^{2}$ averages result in increasing activity, whereas in the ascending phase (1976-1979) the increase in $B_{s}$ causes the activity increase. Thus it seems that variable solar sources are responsible for the varying influence of $v$ and $B_{s}$ on geomagnetic activity.

\subsection{The semiannual variation}

The observation discussed in Sect. 2.1 (see Fig. 1) motivated us to investigate (in addition to the 11-year period) the most pronounced periodic variation; the semiannual wave in geomagnetic activity for timeintervals with high solar wind speed or with a strong IMF- $B_{s}$ component. Although the semiannual activity variation, with maxima around the equinoxes and minima near the solstices, has been known for a long time the interpretation is still controversial. Three mechanisms exist to explain the semiannual variation. First, there was the axial hypothesis, which connects the maxima of the activity with the maximal heliographic latitude of the earth $\left(+7.25^{\circ}\right.$ on 6 September and $-7.25^{\circ}$ on 5 March). This occurs when the position of the earth is favourable for the active solar regions, since it is known that near the solar equator the activity (e.g. flares and sunspots) is minimal (Cortie, 1912). The second mechanism is based on the equinoctial hypothesis (McIntosh, 1959; Boller and Stolov, 1970), suggesting that the interaction between the solar wind and the magnetosphere is maximum when the angle between the solar wind direction and the Earth's dipol is greatest at the equinoxes (21 March and 23 September). The third 
mechanism is based on the reconnection model of interplanetary and geomagnetic field lines, and takes into account the southward component of the IMF with respect to the Earth's magnetic field direction (Russell and McPherron, 1973). This model predicts, on average, maximal southward $B_{s}$ fields and corresponding maximal geomagnetic activity on 5 April and 5 October.

There have been many investigations in recent years to decide which of the models really causes the semiannual wave and, if several mechanisms are effective, which of them is predominant (e.g. Fraser-Smith, 1972; Murayama, 1974; Schreiber, 1981; Green, 1984; Gonzalez et al., 1993). Usually in these studies, the phases of the activity maxima were analysed, but since the maxima predicted by the different models are not very distant from each other and because there are statistical variations in the activity time-series, it is not yet clear which of the mechanisms is the most appropriate. On average, the maxima were found to appear a few days after the equinoxes (25-28 March and 27-30 September, depending on the activity time-series and the kind of analysis); therefore the equinoctial mechanism was often favoured. However, the studies mentioned (and others referenced therein) showed that at least an essential part of the semiannual variation may be explained by the Russell-McPherron (R-M) model .

Whereas the R-M model is based on the southward component of the IMF, the two other mechanisms are more dependent on the solar wind velocity $v$, which increases with the solar latitude (the axial effect) or which, when increased, may generate the Kelvin-Helmholtz instability at the flanks of the magnetosphere (Boller and Stolov, 1970) - the equinoctial effect. Thus, separating the data according to the strength of $B_{S}$ and $v$, one should observe the different influence of $B_{s}$ and $v$ on the activity and perhaps on the semiannual wave.

The hourly values of $v$ and $B_{z}\left(B_{z}\right.$ is the north-south IMF component in solar magnetospheric coordinates) taken from the "OMNI" data base (King, 1977, 1986), as mentioned in Sect. 2.1 for the years 1967-1984, were put into different classes according to the following $v$ and $B_{z}$ intervals:

$$
\begin{aligned}
& 380 \leq v<430,430 \leq v<450,450 \leq v<500,500 \leq v \\
& \quad<600 \mathrm{~km} / \mathrm{s} ; \\
& 3 \leq B_{z}<4,2 \leq B_{z}<3,1.5 \leq B_{z}<2,1.0 \leq B_{z}<1.5,0.5 \\
& \quad \leq B_{z}<1.0, \\
& 0.0 \leq B_{z}<0.5,-0.5 \leq B_{z}<0.0,-1.0 \leq B_{z}<-0.5,-1.5 \\
& \quad \leq B_{z}<-1.0, \\
& -2.0 \leq B_{z}<-1.5,-3.0 \leq B_{z}<-2,-4 \leq B_{z}<-3 \mathrm{nT} .
\end{aligned}
$$

Thus we had $4 \cdot 12=48$ classes with approximately equal numbers of data.

Organizing the ap data in these classes, it is assumed that on average every single ap value is caused by a combination of $B_{z}$ and $v$ values. Although the relationship between ap, $v$ and $B_{z}$ may vary in the development of individual storms, the correlation studies, referred to in Sect. 2.1, indicate that this assumption may be justified for averaging processes. A possible time-lag between $v, B_{z}$ and ap known to be less than $1 \mathrm{~h}$, is neglected, since the ap are three-hourly values.
Next, monthly averages of ap were calculated for every class; e.g. for the class $380 \leq v<430 \mathrm{~km} / \mathrm{s}$ and $3 \leq B_{z}<4 \mathrm{nT}$ the January value was the average of all ap values occurring in January under these solar wind conditions; in the same manner the February, March, etc. ap averages were calculated. Thus we obtained a time-series consisting of 12 monthly averaged ap values with the condition $380 \leq v<430 \mathrm{~km} / \mathrm{s}$ and $3 \leq B_{z}<4$ $\mathrm{nT}$; this yearly ap variation would be seen if during the whole year $v$ and $B_{z}$ were within these limits. For the 48 classes these time-series, each consisting of 12 points, were Fourier transformed to examine the amplitude and phase of the semiannual variation. The results of this procedure were not as expected. Classes with strong southward IMF combined with medium or low solar wind speed resulted in semiannual wave phases with maxima near the equinoxes or earlier, whereas classes with northward IMF fields and high solar wind velocity showed maximal activity according to the R-M mechanism. In Table 1, semiannual amplitudes and phases for some selected classes are listed; the other classes also showed no systematic phase shifts depending on the classes of $v$ or $B_{z}$. We will discuss the semiannual period again in the next section.

\subsection{The 27-day variation}

Investigating the geomagnetic activity variations in the region around the solar rotation period of approximately 27 days, one obtains spectral amplitudes with varying peaks depending on the length of the time-series and the method of analysis (e.g. Fraser-Smith, 1972; Gonzalez et al., 1993). Our Fourier spectral amplitude analysis for periods 30-23 days of the Ap indices 1933-1993 is shown in Fig. 2 together with that of the same but shorter time-series 1933-1983. The rather complex spectra show several different peaks depending on the length of the time-series. Of the four most pronounced peaks at periods of 29.4, 28.9, 27.6 and 27.2 days for the Ap time-interval $1933-1983$ only the 27.2 days period is found in the four greatest amplitudes at periods of 27.2,

\begin{tabular}{|c|c|c|c|c|c|c|}
\hline \multicolumn{2}{|c|}{$\begin{array}{l}v \text { interval } \\
(\mathrm{km} / \mathrm{s})\end{array}$} & \multicolumn{2}{|c|}{$\begin{array}{l}B \text { interval } \\
(\mathrm{nT})\end{array}$} & \multirow{2}{*}{$\begin{array}{l}\begin{array}{l}\text { amplitude } \\
\text { (ap-units) }\end{array} \\
1.7\end{array}$} & \multirow{2}{*}{$\begin{array}{l}\text { date of } \\
\text { max. } \\
1 \text { April }\end{array}$} & \multirow{2}{*}{$\begin{array}{l}\text { remarks } \\
\text { expected }\end{array}$} \\
\hline 380 & 430 & -3.0 & -2.0 & & & \\
\hline 380 & 430 & 2.0 & 3.0 & 0.7 & 5 April & unexpected \\
\hline 430 & 450 & -1.5 & -1.0 & 0.7 & 1 April & expected \\
\hline 430 & 450 & 1.0 & 1.5 & 0.7 & 14 March & expected \\
\hline 450 & 500 & -1.5 & -1.0 & 1.7 & 6 April & expected \\
\hline 450 & 500 & 1.0 & 1.5 & 1.5 & 1 April & unexpected \\
\hline 500 & 600 & -1.5 & -1.0 & 1.8 & 22 March & unexpected \\
\hline 500 & 600 & 1.0 & 1.5 & 1.4 & 12 April & unexpected \\
\hline
\end{tabular}
26.7, 25.8 and 24.0 days for the 1933-1993 interval. The respective amplitudes, and several more, exceed the rms error of $0.2 \mathrm{Ap}$ units; the mean spectral amplitude in that period range is $0.35 \mathrm{Ap}$ units. It should be noted

Table 1. Examples of amplitudes and phases of the semiannual wave of ap for different solar wind speed $v$ and IMF $B_{z}$ intervals 


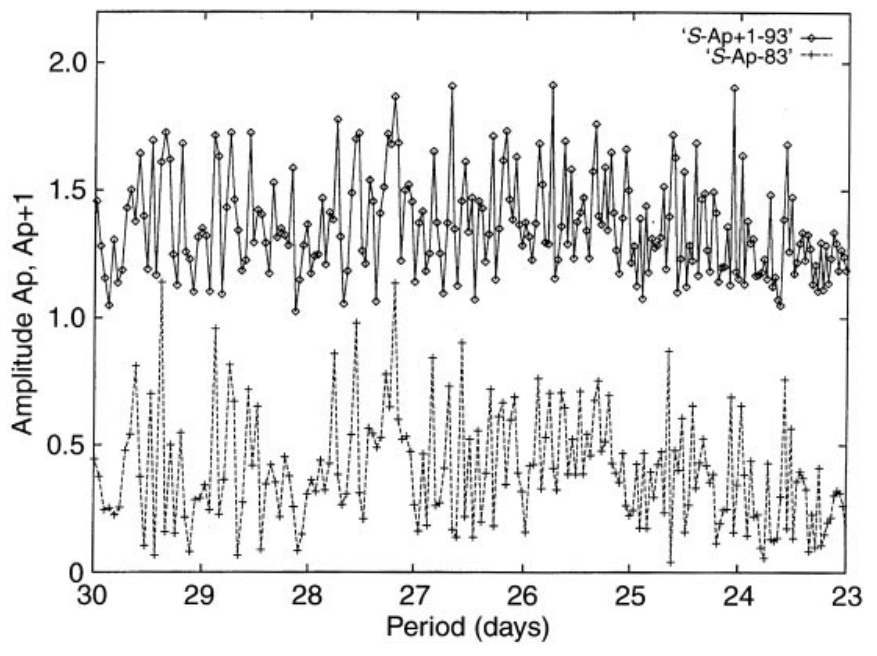

Fig. 2. Fourier spectral amplitudes for the period interval 30-23 days of the Ap time-series 1933-1993 (S-Ap + 1-93) and 1933-1983 (S-Ap83), showing maximal peaks for different periodicities, depending on the length of the time-series. In the upper curve one Ap unit was added to the Fourier amplitudes $S$-Apr $+1-93$ to separate the two spectra

that an additional light smoothing of the spectra did not change their general features.

The rather spurious nature of the Ap Fourier spectrum around the 27-day period is due to non-persistent periodicities of the activity generating solar sources, for instance the development of coronal holes and their disappearance, whereas new holes appear at other solar longitudes. Therefore our Fourier spectral analysis is not the appropriate method to analyse the 27-day period of the longer Ap time-series. However, the power spectral analysis as applied by Gonzalez et al. (1993) also resulted in rather complex spectra with several significant peaks, of which the greatest near 27.2 days and that near 29.4 days are also seen in our Fourier spectra. On the other hand, the rotation period of the solar sources is known to vary between 26 and 29 days depending of the phase of the solar cycle. Therefore, to investigate the 27-day period variation in detail, one should split up the data into shorter time-intervals where persistence may be expected. First attempts to separate longer (28-29 days) and shorter (26-27 days) solar rotation periods during the solar cycles, investigating Ap in the 26-29 day period interval for different phases of the sunspot cycle or for different ranges of sunspot numbers, gave the same unclear results, as seen in the spectra of Fig. 2.

Finally, a discriminating feature that splits the data was found in the varying recurrence period of the sector structure of the interplanetary magnetic field with IMF directions towards and away from the sun, which in reality reflects the wavy structure of the heliospheric current sheet (e.g. Svalgaard and Wilcox, 1975; Hoeksema et al., 1983). The recurrence period of this sector structure is known to reflect on a global scale the average solar rotation period. It also has been shown that "a basic variability of the sector structure becomes apparent with characteristic times on the order of a few years" (Gonzalez and Gonzalez, 1987) and that the fundamental period changes from 28 to 27 days according to the phase of the solar cycle. For our analysis we used the IMF polarity inferred from the daily variation of ap, which was found to give reasonable results for statistical purposes (Schreiber, 1978). In Fig. 3 the sector structure, inferred using exactly this method for the years 1932.5-1983.5 (i.e. for sunspot cycles 17-21), is shown in the upper part, together with monthly averages of Ap and the sunspot number $R$ below. The sector structure is depicted with vertical (with respect to the time-axis) lines of 27 days' length, repeated three times, so that the structure may be better recognized. Here black indicates IMF polarity towards the sun and white indicates IMF direction away from the sun. If the signatures of the symbolic structure are aligned parallel to the time-axis (e.g. the years 50-53, 62-64), one sees a 27-day recurrence period. If the structure is oblique to the time-axis, longer periods exist in the sector structure (e.g. the years 55-56, 78-79). This structure was split into intervals of 2 years' (exactly $27 \cdot 27=729$ days) length and, with the superposed epoch analysis (e.g. Chapman and Bartels, 1940), we looked for statistically significant periods of $29.0,28.5,28.0, \ldots, 26.5,26.0$ days. The data samples of 2 years' length were shifted by 0.5 years so that the intervals 1932.5-1934.5, 1933.0-1935.0, 1935.5$1937.5, \ldots, 1981.5-1983.5$ were analysed separately. Those intervals for which significant periods were found after this procedure are indicated with rectangles in the middle of Fig. 3, white rectangles for a 27-day period and hatched rectangles for 28.0- or 28.5-day periods. No other statistically significant periods were found. The sector structure (not the activity itself) generally shows significant recurrence periods of 27 days before and during the sunspot minima, 28-28.5 days after the sunspot minima, and during some 2-year intervals after the maxima. A similar solar-cycle-dependent variation of the recurrence period of the IMF sector structure was found by Svalgaard (1972) using his classification of IMF towards and away polarity based on diurnal magnetic variations at near-pole magnetic observatories.

For all those two-year intervals with significant periods of the IMF sector structure, the Fourier spectral amplitudes of Ap were computed (spectra of 730 or 731 continuous daily Ap values). The spectra were put into two classes according to the period of 27 or 28-28.5 days of the IMF sector structure. These averaged Ap spectra of periods in the range of 42 to 8 days are shown in Fig. 4. Two different periodicities, together with their higher harmonics, are clearly seen for the two groups with peaks at 27.0, 13.8 and 9.0 days for the years before solar minimum (S-AMIN) and at 28.6, 14.9 and 9.9 days for years after solar minimum (S-PMIN); the amplitudes for the higher harmonics, although well above the noise level, are not so distinct. These two periods of geomagnetic activity (especially the 27-day period before the solar sunspot cycle minimum) have been known for some time (e.g. Fraser-Smith, 1972; Sargent, 1986). Whereas the phases of the averaged spectral amplitudes are insignificant because of the mentioned phase shifts of the solar sources, a strong relation is seen between the 


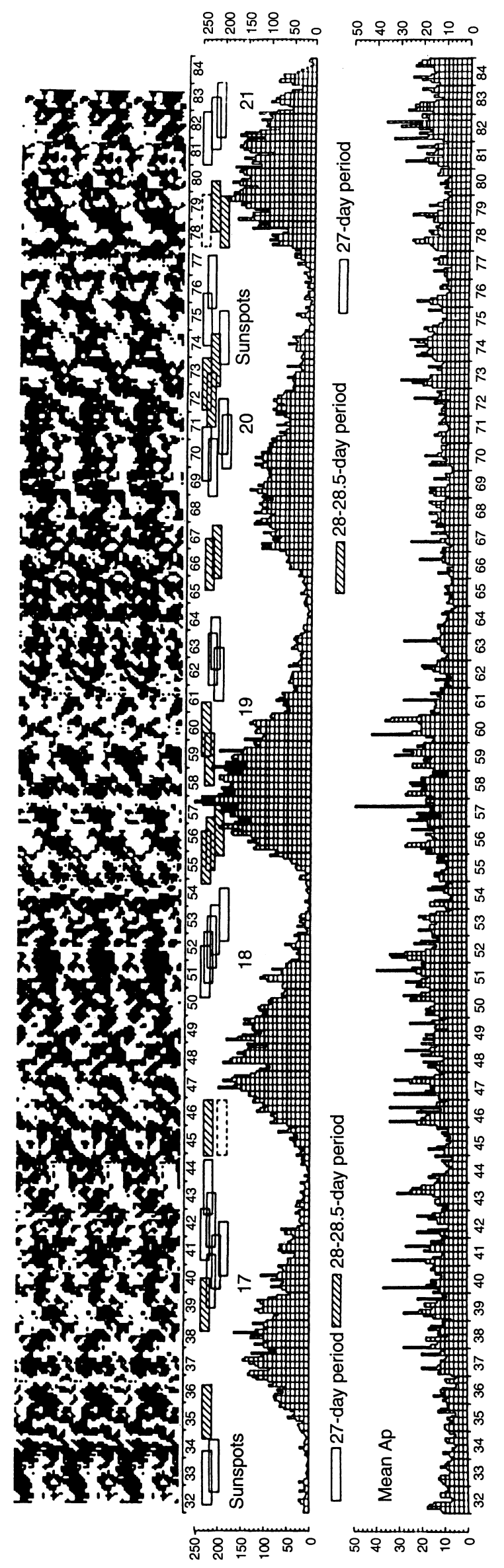

Fig. 3. Monthly means of Ap and of sunspot numbers for cycles 1721, years 1932-1984, in the bottom and central parts. The upper part shows the sector structure of the interplanetary magnetic field IMF as inferred from diurnal ap variations for the years 1932.5-1983.5; black areas for IMF direction towards the sun and white areas for IMF direction away from the sun are drawn vertically with respect to the time-scale for 27.0 days of Bartels rotations, the structure being repeated three times. The rectangular white, hatched boxes indicate intervals where significant 27- or 28-28.5-day periods were found in the 2-year data sets

recurrence periods of the IMF sector structure and geomagnetic activity.

More interesting is the fourth harmonic of the 2-year fundamental period of the two averaged spectra of Fig. 4, representing the semiannual wave. The amplitudes and phases of these waves are shown in the harmonic dial (Bartels, 1935) of Fig. 5. Two significant different vectors are seen, one, AMIN, of length $3.61 \mathrm{Ap}$ units pointing to the equinox (20 March) for years before sunspot minimum and the other, PMIN, of length $2.89 \mathrm{Ap}$ units pointing to the phase predicted by the R-M model (2 April). The average semiannual wave of the whole time-series 1933-1993, with amplitude of $2.78 \mathrm{Ap}$ units, points to $27 \mathrm{March}$. Thus, we may have found time-intervals during the solar cycle when geomagnetic activity is more influenced by a southward IMF, generating a semiannual wave according to the R$\mathrm{M}$ model and intervals dominated by the solar wind velocity and resulting in semiannual waves according to the equinoctial (or also axial) mechanism.

\subsection{The universal time variation of ap}

The Russell-McPherron mechanism which, according to the foregoing section, should be most effective after

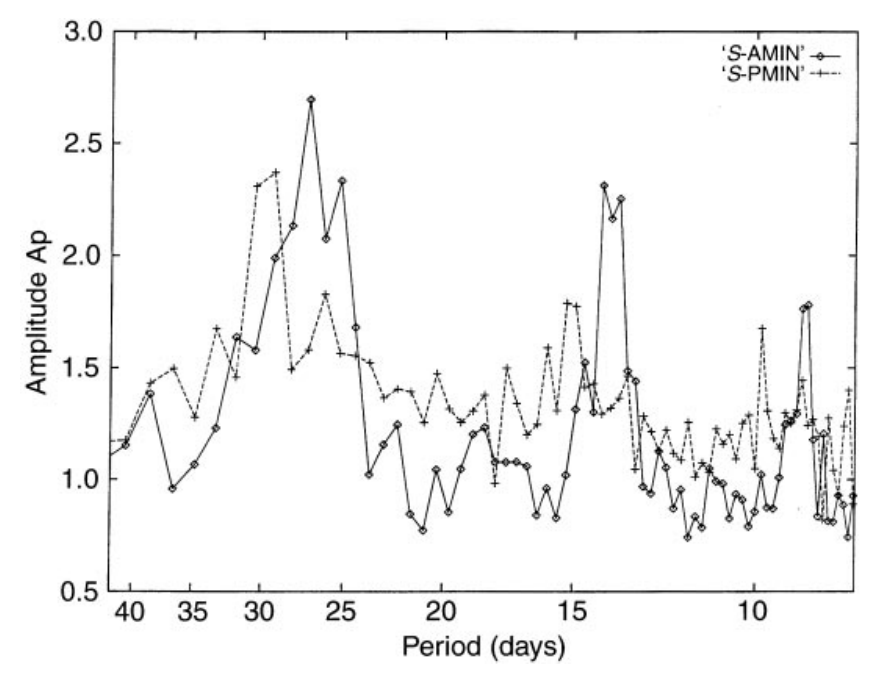

Fig. 4. Average spectral amplitude of Ap for years before sunspot minimum with a 27 -day period of the IMF sector structure, S-AMIN, and of years after sunspot minimum with 28-28.5-day recurrence period of the IMF sector structure, S-PMIN. The spectral Ap amplitudes $a_{n}$ are smoothed with $a_{n}=0.25\left(a_{n-1}^{+} 2 a_{n}^{\prime+} a_{n+1}^{\prime}\right)$, where $a_{n}^{\prime}$ are the original Fourier amplitudes. Notice the $\log$-scale of the abszissa in the period range $42-8$ days 


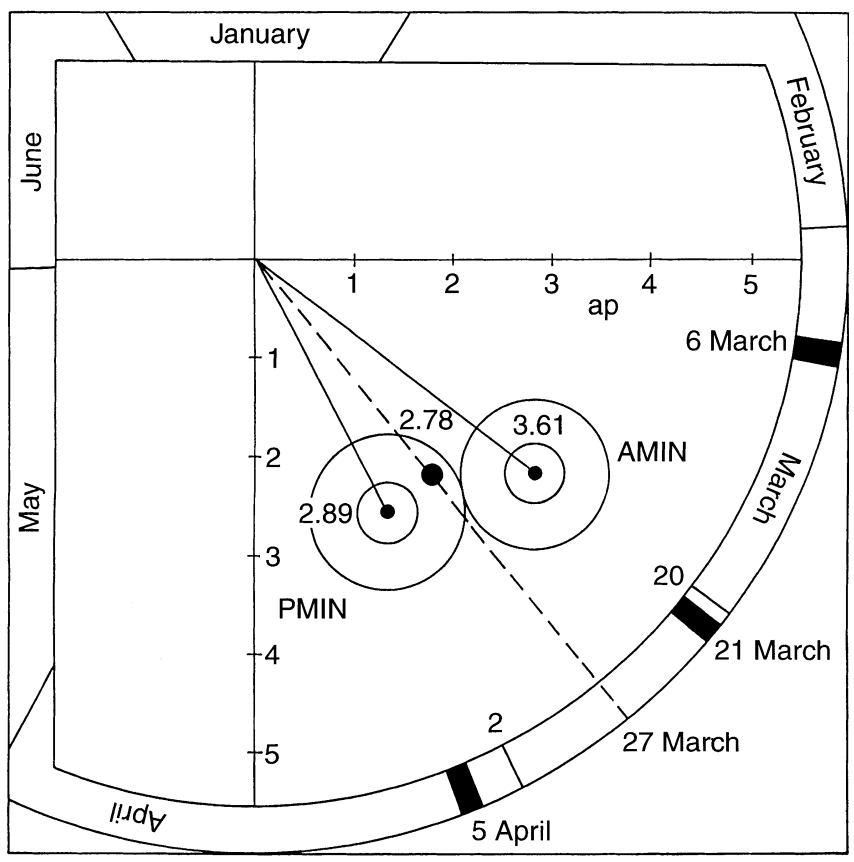

Fig. 5. Harmonic dial showing the amplitude and phase of maximum for the semiannual wave of the two averaged spectra shown in Fig. 4, AMIN and PMIN before and after sunspot minimum, respectively, and the average semiannual wave of Ap for the years 1933-1993. The large circles denote the probable errors for the single values, the small circles denote the errors of the averages

sunspot minima, also predicts the season-dependent daily UT variations (Russell and McPherron, 1973). These are quite different from daily UT variations predicted by the equinoctial mechanism (e.g. Boller and Stolov, 1970) which is expected to be effective for years before sunspot minimum. Several investigations of the diurnal UT variations of geomagnetic activity, using the am indices (Mayaud, 1980), have shown no clear results regarding the R-M or the equinoctial hypothesis (Russell and McPherron, 1973; Berthelier, 1976; Russell, 1989; Orlando et al., 1995), suggesting that both mechanisms may cause geomagnetic activity.

Here we examine the UT variation of the ap indices [although according to their definition they should not have average UT variation, and if such average variations were found (as it is the case), they are due to the imperfection of the ap computing procedure (Siebert, 1971)]. However, calculating the diurnal ap variations for periods with different generating processes, resulting in different amplitudes and phase for the diurnal UT variation, the difference in the theoretical generating mechanisms should also be seen in the difference of the respective diurnal ap variations.

The most distinctive different diurnal ap variations of the R-M effect and the equinoctial effect are predicted for the vernal and autumnal months. As the diurnal ap variation of March minus diurnal ap variation of September after the R-M hypothesis results in a 1-day period with maximum at 22:30 UT and minimum at 10:30 UT, the respective difference of the daily variations almost vanishes for the equinoctial hypothesis (Russell and McPherron, 1973, Fig. 11).
In Fig. 6a the difference of the diurnal variations of March and September of the southward IMF component $B_{s}$ after the R-M model $\left(-B_{s}\right.$ (mar) $+B_{s}$ (sep)) is drawn together with the respective difference curve for ap (ap (mar) - ap (sep)). The ap curve is computed from data of the years 1933-1993, ap (mar), including the months March and April, and ap (sep) including the months September and October. The similarity of the two curves of Fig. 6a suggests a major contribution of the R-M effect to the average diurnal UT variation of ap. The amplitude of the variation with maximum and minimum at about $22: 30$ and 10:30 UT is roughly 1.5 ap units.

In Fig. 6b the analogue differences of diurnal ap curves for the two groups of years of Sect. 2.3, namely the years before sunspot minimum (curve apamin), and the years after sunspot minimum (curve appmin), are
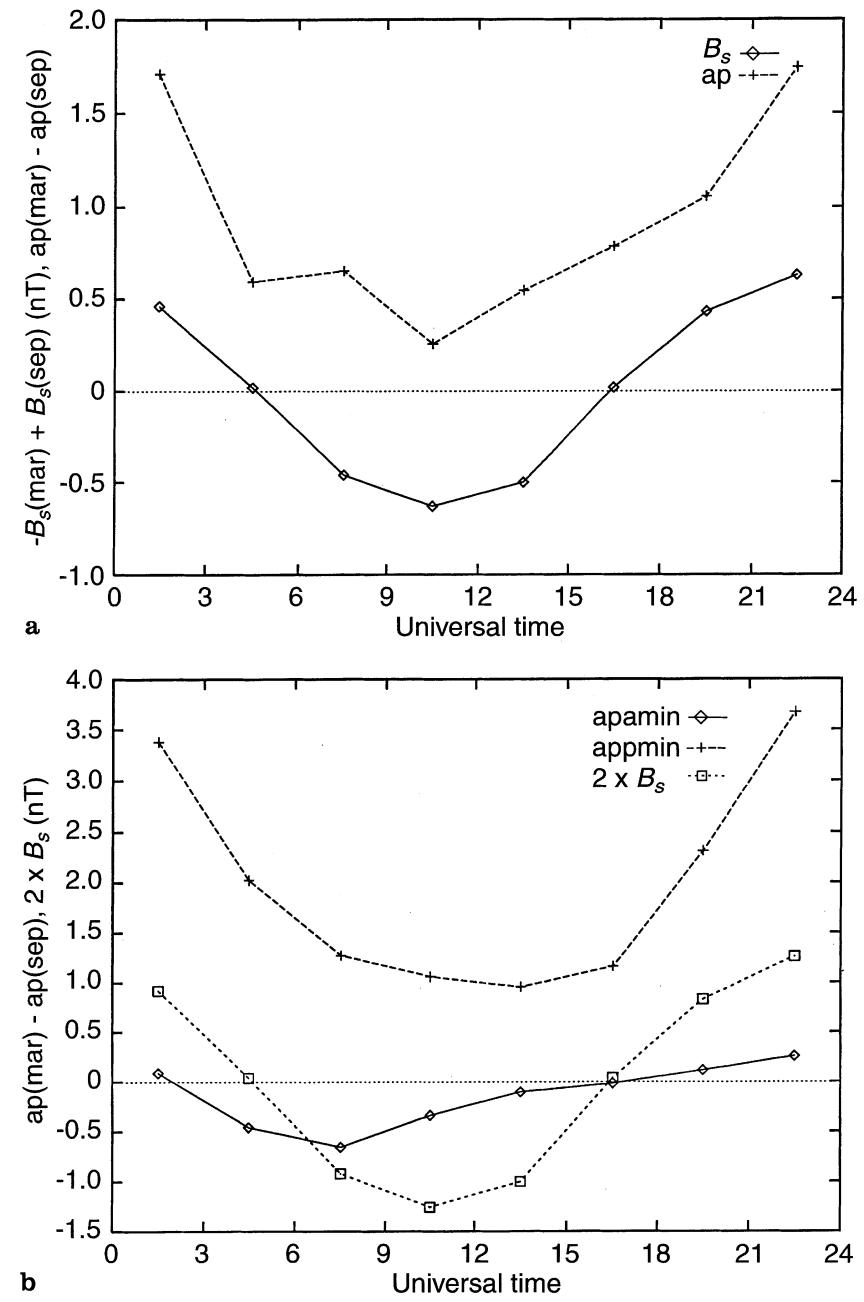

Fig. 6. a Difference of the March and September UT variations of the IMF southward component $B_{s}$ as predicted by the Russell-McPherron effect $-B_{s}$ (mar) $+B_{s}$ (sep) and the respective difference of the UT variations of ap (years 1933-1993) ap (mar) - ap (sep); the sign of the $B_{s}$ curve was chosen so that the minimum at 10:30 UT matches the minimum of the ap curve. b Difference of the vernal and autumnal UT variations of ap for the same groups of years before (apamin) and after (appmin) sunspot minimum as in Figs 4 and 5 together with the UT variation of $B_{s}$ of Fig. 6a multiplied by a factor of 2 
drawn together with the $B_{s}$ curve of Fig. 6a doubled $\left(2 * B_{s}\right)$. The diurnal amplitude of the appmin curve is now enhanced compared to the ap curve of Fig. 6 a (maximal minus minimal value is about 2.3 ap units), whereas the amplitude of the apamin curve is decreased compared to the ap curve in Fig. 6a (maximal minus minimal value is about 0.9 ap units). Thus, even in the diurnal UT variation (as in the semiannual), the influence of the equinoctial effect is enhanced (the R$M$ effect decreased) during years before sunspot minimum and the R-M effect is enhanced (the equinoctial effect decreased) during years after sunspot minimum.

\section{Discussion and conclusion}

Yearly averages of the geomagnetic Ap indices are well correlated with the respective averages of the solar wind parameter $v^{2} B_{s}$. However, separating $v^{2}$ and $B_{s}$, we see varying influence of $v^{2}$ and $B_{s}$ in the course of a solar cycle, especially before and after sunspot minimum. Applying this observation to the investigation of the semiannual wave of geomagnetic activity in order to decide whether the equinoctial mechanism is effective when the solar wind speed $v$ is high and IMF- $B_{z}$ component is directed further northwards (during these conditions also the axial mechanism might be effective) or whether the R-M mechanism is active when $v$ is decreased and $B_{z}$ is directed southwards, the results were inconclusive. However, when the data were divided according to the significant 27- or 28-28.5-day periodicity of the IMF sector structure, representing the wavy heliospheric current sheet, the same 27- and 28-28.5-day modulation periods with their higher harmonics, before and after sunspot minimum, respectively, were found in the geomagnetic activity. In addition, we found different phases in the semiannual variation of the activity, suggesting that the equinoctial model applies more during years before sunspot minimum when a 27-day modulation period is seen, and that the R-M mechanism is more effective after sunspot minimum when the 28 28.5-day modulation period is observed. No evidence was found for the axial mechanism.

Since the equinoctial and R-M model theoretically predict different diurnal UT variations, we investigated the diurnal waves for the two sets of data. The results of this analysis were consistent with those for the semiannual wave, indicating that the R-M and equinoctial mechanisms have varying importance on geomagnetic activity during the course of the solar activity cycle.

Altogether, our analysis of the yearly averages of Ap and the solar wind parameters $v^{2}$ and $B_{s}$, as well as the spectral analysis of the semiannual and 27- or 28-28.5day periodicities and the diurnal UT variations of ap, give a consistent picture as to the solar sources of the observed activity variations. A rough and oversimplified sketch would be the following: before the solar cycle minimum, long-lived coronal holes with higher solar wind velocities extend to low solar latitudes and to the solar equator, the interplanetary magnetic field shows only slight north-south fluctuations, and the flat helio- spheric current sheet is near the solar equatorial plane (e.g. Hoeksema et al., 1983). Therefore, geomagnetic activity is generated more by the magnitude of the solar wind velocity, and less by the southward IMF field $B_{s}$. After a solar cycle minimum (and also after solar maxima), less coronal holes exist reaching the solar equator, whereas the heliospheric current sheet and accordingly the IMF exhibit strong north-south fluctuations (e.g. Hoeksema et al., 1983). Therefore $B_{s}$ is stronger on average, and the reconnection process at the dayside magnetopause results in periodicities of geomagnetic activity with phases according to the R-M model. This simplified view is consistent with the comprehensive description of geomagnetic activity and its relation to the solar sources during the declining phase of the solar cycle given by Tsurutani et al. (1995).

Acknowledgements. The author wishes to thank M. Siebert for helpful discussions and M. Heimpel for his assistance during the preparation of the manuscript. He also acknowledges the critical comments of one referee.

Topical Editor D. Alcaydé thanks A.L. Cluá de Gonzalez for her help in evaluating this paper.

\section{References}

Arnoldy, R. L., Signature of the interplanetary medium for substorms, J. Geophys. Res., 76, 5189-5201, 1971.

Bartels, J., Random fluctuation, persistence and quasi-persistence in geophysical and cosmical periodicities, Terr. Magn. Atmos. Electr., 40, 1-60, 1935.

Berthelier, A., Influence of the polarity of the interplanetary magnetic field on the annual and the diurnal variations of magnetic activity, J. Geophys. Res., 81, 4546-4552, 1976.

Boller, B. R., and H. L. Stolov, Kelvin-Helmholtz instability and the semiannual variation of geomagnetic activity, J. Geophys. Res., 75, 6073-6084, 1970.

Chapman, S., and J. Bartels, Geomagnetism, Oxford University Press, New York, 1940.

Cortie, A. L., Sunspots and terrestrial magnetic phenomena 18981911, Mon. Not. R. Astron. Soc., 73, 52-60, 1912.

Crooker, N. U., and E.W. Cliver, Postmodern view of M-regions, $J$. Geophys. Res., 99, 23383-23390, 1994.

Crooker, N. U., and C.L. Sicoe, The effect of the solar wind on the terrestrial environment, in Physics of the sun, vol. III, Eds. P.A. Sturrock, T.E. Holzer, D.M. Mihalas, and R.K. Ulrich, D. Reidel, Dordrecht, pp. 193-249, 1986.

Dungey, J. W., Interplanetary magnetic fields and the auroral zones, Planet. Res. Lett., 6, 47-48, 1961.

Dungey, J. W., Memories, maxims and motives, J. Geophys. Res., 99, 19189-19197, 1994.

Fraser-Smith, A. C., Spectrum of the geomagnetic activity Index Ap, J. Geophys. Res., 77, 4209-4220, 1972.

Gonzalez, A. L. C., and D. W. Gonzalez, Periodicities in the interplanetary magnetic field polarity, J. Geophys. Res., 92, 4357-4375, 1987.

Gonzalez, W. D., A. L. C. Gonzalez, and B. T. Tsurutani, Dualpeak solar cycle distribution of intense geomagnetic storms, Planet. Space Sci., 38, 181-187, 1990.

Gonzalez, A. L. C., W. D. Gonzalez, S. L. G. Dutra, and B. T. Tsurutani, Periodic variation in the geomagnetic activity: a study based on the Ap Index, J. Geophys. Res., 98, 9215-9231, 1993.

Green, C. A., The semiannual variation in the magnetic activity indices Aa and Ap, Planet. Space Sci., 32, 297-305, 1984. 
Hirshberg, J., and D. S. Colburn, The interplanetary magnetic field and geomagnetic variations - a unified view, Planet. Space Sci., 17, 1183-1206, 1969.

Hoeksema, J. T., J. M. Wilcox, and P.H. Scherrer, The structure of the heliospheric current sheet: 1978-1982, J. Geophys. Res., 88, 9910-9918, 1983

King, J. H., Interplanetary medium data book, Rep. NSSCD 77-04, NASA, Goddard Space Flight Cent., Greenbelt, Md., 1977.

King, J. H., Interplanetary medium data book - supplement 3, 1977 1985, Rep NSSDC 86-04, NASA, Goddard Space Flight Cent., Greenbelt, Md., 1986.

Lindsay, G. M., C. T. Russell, and J. G. Luhmann, Coronal mass ejection and stream interaction region characteristics and their potential geomagnetic effectiveness, J. Geophys. Res., 100, 16999-17013, 1995.

Mayaud, P. N., Derivation, meaning, and use of geomagnetic indices, Geophys. Monogr 22, AGU, Washington, D.C., 1980

McIntosh, D. H., On the annual variation of magnetic disturbances, Philos. Trans. R. Soc. London Ser. A, 251, 525-552, 1959.

Murayama, T., Origin of the semiannual variation of geomagnetic Kp indices, J. Geophys. Res., 79, 297-300, 1974.

Orlando, M., G. Moreno, M. Parisi, and M. Storini, Diurnal modulation of the geomagnetic activity induced by the southward component of the interplanetary magnetic field, $J$. Geophys. Res., 100, 19565-19570, 1995.

Petschek, H. E., Magnetic field annihilation, in AAS-NASA Symposium on the physics of solar flares, Ed. W.N. Hess, NASA Spec. Publ., Sp-50, p. 425, 1964.
Russell, C. T., The universal time variation of geomagnetic activity, J. Geophys. Res. Lett., 16, 555-558, 1989.

Russell, C. T., and R. L. McPherron, Semiannual variation of geomagnetic activity, J. Geophys. Res., 78, 92-108, 1973.

Sargent, H. H., III, The 27-day recurrence index, in Solar windmagnetosphere coupling, Eds. Y. Kamide and J.A. Slavin, Terra Scientific, Tokyo, pp. 143-148, 1986.

Schreiber, H., On the possibility of inferring the interplanetary sector structure from daily variations of geomagnetic ap indices, Planet. Space Sci., 26, 767-775, 1978.

Schreiber, H., Correlation of geomagnetic activity indices ap with the solar wind speed and the southward interplanetary magnetic field, J. Geophys., 49, 169-175, 1981.

Siebert, M., Maßzahlen der erdmagnetischen Aktivität, in Handbuch der Physik Bd. 49/3 (Geophysik 3/3), Ed. S. Flügge, Springer, Berlin, Heidelberg, New York, pp. 206-275, 1971.

Svalgaard, L., Interplanetary magnetic sector structure, 1926-1971, J. Geophys. Res., 77, 4027-4034, 1972.

Svalgaard, L., and J. M. Wilcox, Long-term evolution of solar sector structure, Solar Phys., 41, 461-475, 1975.

Tsurutani, B. T., W. D. Gonzalez, A. L. C. Gonzalez, F. Tang, J. K. Arballo, and M. Okada, Interplanetary origin of geomagnetic activity in the declining phase of the solar cycle, J. Geophys. Res., 100, 21717-21733, 1995.

Vasilyunas, V. M., Theoretical models of magnetic field line merging I., Rev. Geophys. Space Phys., 13, 303-336, 1975. 